\title{
Joint distribution models in fast-moving consumer goods wholesale enterprise: Comparative analysis and a case study
}

\author{
Wang, L. ${ }^{a}$, Chen, X.Y. ${ }^{a,}{ }^{*}$, Zhang, H. ${ }^{a, b}$ \\ ${ }^{a}$ School of E-Business and Logistics, Beijing Technology and Business University, Beijing, P.R. China \\ ${ }^{b}$ Beijing Food Safety Research Base, Beijing, P.R. China
}

\begin{abstract}
A B S T R A C T
Joint distribution means multiple clients were provided distribution services together by only one third-party logistics company. It is a unified plan and implementation used in distribution centres and a distribution activity implemented by multiple consortia. Many problems in distribution can be solved through the joint use of distribution warehouse, vehicles and reasonable logistics business, so as to optimize the overall logistics node and route arrangement. This paper mainly discusses the model of joint distribution of fast moving consumer goods, proposes three types of the optimization model of joint distribution system with Chaopi as an example. We draw the conclusion that Chaopi Trading Co., Ltd. is a joint distribution system optimization business model. This paper puts forward several basic distribution models and analyzes them in combination with practical applications, which has strong practical significance. Although the development of public distribution in China is not very fast, it is an inevitable trend. Through the efforts and explorations of the governments of various countries, there will be more and more choices of public distribution models.
\end{abstract}

\author{
ARTICLE INFO \\ Keywords: \\ Logistics; \\ Joint distribution; \\ Wholesale enterprise; \\ Fast-moving consumer goods; \\ Distribution models; \\ Optimization \\ *Corresponding author: \\ 845190504@qq.com \\ (Chen, X.Y.) \\ Article history: \\ Received 27 March 2021 \\ Revised 23 May 201 \\ Accepted 15 June 2021

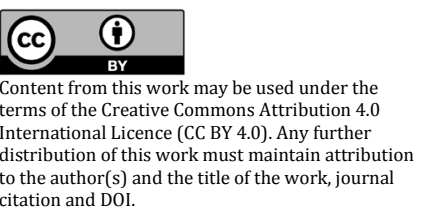

\section{References}

[1] Vieira, J.G.V., Fransoo, J.C. (2015). How logistics performance of freight operators is affected by urban freight distribution issues, Transport Policy, Vol. 44, 37-47, doi: 10.1016/j.tranpol.2015.06.007.

[2] Gómez, S.C.G., Cruz-Reyes, L., González, B.J.J., Fraire, H.H.J., Pazos, R.R.A., Martínez, P.J.J. (2014). Ant colony system with characterization-based heuristics for a bottled-products distribution logistics system, Journal of Computational and Applied Mathematics, Vol. 259, Part B, 965-977, doi: 10.1016/i.cam.2013.10.035.

[3] Wu, Y.-C.J., Huang, S.K. (2013). Making on-line logistics training sustainable through e-learning, Computers in Human Behavior, Vol. 29, No. 2, 323-328, doi: 10.1016/j.chb.2012.07.027.

[4] Liu, Y. (2015). An empirical study on customers' satisfaction of third-party logistics services (3PLS), In: Proceedings of the 2015 International Conference on Education, Management and Computing Technology, Atlantis Press, 1361-1365, doi: 10.2991/icemct-15.2015.282.

[5] Cabigiosu, A., Campagnolo, D., Furlan, A., Costa, G. (2015). Modularity in KIBS: The case of third-party logistics service providers, Industry and Innovation, Vol. 22, No. 2, 126-146, doi: 10.1080/13662716.2015.1023012.

[6] Lopes, H.S., Lima, R.S., Leal, F. (2020). Simulation project for logistics of Brazilian soybean exportation, International Journal of Simulation Modelling, Vol. 19, No. 4, 571-582, doi: 10.2507/IJSIMM19-4-529.

[7] Aguezzoul, A. (2014). Third-party logistics selection problem: A literature review on criteria and methods, Omega, Vol. 49, 69-78, doi: 10.1016/i.omega.2014.05.009. 
[8] Shi, Y., Zhang, A., Arthanari, T., Liu, Y. (2016). Third-party purchase: An empirical study of Chinese third-party logistics users, International Journal of Operations \& Production Management, Vol. 36, No. 3, 286-307, doi: 10.1108/IJOPM-11-2014-0569.

[9] Moutaoukil, A., Neubert, G., Derrouiche, R. (2015). Urban freight distribution: The impact of delivery time on sustainability, IFAC-PapersOnLine, Vol. 48, No. 3, 2368-2373, doi: 10.1016/j.ifacol.2015.06.442.

[10] Freile, A.J., Mula, J., Campuzano-Bolarin, F. (2020). Integrating inventory and transport capacity planning in a food supply chain, International Journal of Simulation Modelling, Vol. 19, No. 3, 434-445, doi: 10.2507/IJSIMM193-523.

[11] $\mathrm{Hu}, \mathrm{H} ., \mathrm{Wu}, \mathrm{Q}$., Zhang, Z., Han, S. (2019). Effect of the manufacturer quality inspection policy on the supply chain decision-making and profits, Advances in Production Engineering \& Management, Vol. 14, No. 4, 472-482, doi: 10.14743/apem2019.4.342.

[12] Trappey, A.J.C., Trappey, C.V., Govindarajan, U.H., Chuang, A.C., Sun, J.J. (2017). A review of essential standards and patent landscapes for the internet of things: A key enabler for Industry 4.0, Advanced Engineering Informatics, Vol. 33, 208-229, doi: 10.1016/j.aei.2016.11.007.

[13] Li, H.-Y., Xu, W., Cui, Y., Wang, Z., Xiao, M., Sun, Z.-X. (2020). Preventive maintenance decision model of urban transportation system equipment based on multi-control units, IEEE Access, Vol. 8, 15851-15869, doi: 10.1109/ ACCESS.2019.2961433.

[14] Awaga, A.L., Xu, W., Liu, L., Zhang, Y. (2020). Evolutionary game of green manufacturing mode of enterprises under the influence of government reward and punishment, Advances in Production Engineering \& Management, Vol. 15, No. 4, 416-430, doi: 10.14743/apem2020.4.375.

[15] De Marco, A., Cagliano, A.C., Mangano, G., Perfetti, F. (2014). Factor influencing logistics service providers efficiency in urban distribution systems, Transportation Research Procedia, Vol. 3, 499-507, doi: 10.1016/i.trpro. 2014.10.031. 
APEM
Advances in Production Engineering \& Management

Letnik 16 | Številka 2 | Junij 2021 | Strani 212-222

https://doi.org/10.14743/apem2021.2.395
ISSN 1854-6250

Spletna stran: apem-journal.org Izvirni znanstveni članek

\title{
Modeli skupne distribucije blaga široke potrošnje $v$ veleprodajnem podjetju: Primerjalna analiza in študija primera
}

\author{
Wang, L. ${ }^{a}$, Chen, X.Y. ${ }^{a,}{ }^{*}$, Zhang, H. ${ }^{\text {a,b }}$ \\ aSchool of E-Business and Logistics, Beijing Technology and Business University, Beijing, P.R. China \\ ${ }^{b}$ Beijing Food Safety Research Base, Beijing, P.R. China
}

\section{POVZETEK}

Skupna distribucija pomeni, da več strankam distribucijske storitve zagotavlja samo eno zunanje logistično podjetje. Gre za enoten načrt in izvedbo, ki se uporabljata v distribucijskih centrih, in distribucijsko dejavnost, ki jo uporablja več konzorcijev. Številne težave pri distribuciji je mogoče rešiti s skupno uporabo distribucijskega skladišča, vozil in razumnega logističnega poslovanja. Na ta način se optimizira celotno logistično vozlišče in se razporejajo poti. Prispevek obravnava model skupne distribucije blaga široke potrošnje na primeru podjetja Chaopi in predlaga tri vrste optimizacijskega modela skupnega distribucijskega sistema. Sklenemo lahko, da je poslovni model podjetja Chaopi Trading Co., Ltd. optimiziran skupni distribucijski sistem. Ta članek predstavlja več osnovnih distribucijskih modelov in jih analizira v kombinaciji s praktičnimi aplikacijami, kar ima velik praktični pomen. Čeprav razvoj javne distribucije na Kitajskem ni zelo hiter, je to neizogiben trend. S prizadevanji in vladnimi raziskavami različnih držav bo na izbiro vedno več modelov javne distribucije.

\section{PODATKI O ČLANKU}

Ključne besede:

Logistika;

Skupna distribucija;

Veleprodajno podjetje;

Blago široke potrošnje;

Distribucijski modeli;

Optimizacija

* Kontaktna oseba:

845190504@qq.com

(Chen, X.Y.)

Zgodovina članka:

Prejet 27. marca 2021

Popravljen 23. maja 2021

Sprejet 15. junija 2021 